\title{
THE FORMATION MECHANISM OF GAS GIANTS ON WIDE ORBITS
}

\author{
Sarah E. Dodson-Robinson ${ }^{1,4}$, Dimitri Veras ${ }^{2}$, Eric B. Ford ${ }^{2}$, and C. A. Beichman ${ }^{3}$ \\ ${ }^{1}$ Astronomy Department, University of Texas, 1 University Station C1400, Austin, TX 78712, USA; sdr@ astro.as.utexas.edu \\ ${ }^{2}$ Astronomy Department, University of Florida, 211 Bryant Space Sciences Center, Gainesville, FL 32111, USA \\ ${ }^{3}$ NASA Exoplanet Science Institute, California Institute of Technology, 770 S. Wilson Ave, Pasadena, CA 91125, USA \\ Received 2009 July 29; accepted 2009 October 19; published 2009 November 18
}

\begin{abstract}
The recent discoveries of massive planets on ultra-wide orbits of HR 8799 and Fomalhaut present a new challenge for planet formation theorists. Our goal is to figure out which of three giant planet formation mechanismscore accretion (with or without migration), scattering from the inner disk, or gravitational instability-could be responsible for Fomalhaut b, HR 8799 b, c and d, and similar planets discovered in the future. This paper presents the results of numerical experiments comparing the long-period planet formation efficiency of each possible mechanism in model A star, G star, and M star disks. First, a simple core accretion simulation shows that planet cores forming beyond 35 AU cannot reach critical mass, even under the most favorable conditions one can construct. Second, a set of $N$-body simulations demonstrates that planet-planet scattering does not create stable, wide-orbit systems such as HR 8799. Finally, a linear stability analysis verifies previous work showing that global spiral instabilities naturally arise in high-mass disks. We conclude that massive gas giants on stable orbits with semimajor axes a 35 AU form by gravitational instability in the disk. We recommend that observers examine the planet detection rate as a function of stellar age, controlling for the planets' dimming with time. Any age trend would indicate that planets on wide orbits are transient relics of scattering from the inner disk. If planet detection rate is found to be independent of stellar age, it would confirm our prediction that gravitational instability is the dominant mode of producing detectable planets on wide orbits. We also predict that the occurrence ratio of long-period to short-period gas giants should be highest for M dwarfs due to the inefficiency of core accretion and the expected small fragment mass $\left(\sim 10 M_{\text {Jup }}\right)$ in their disks.
\end{abstract}

Key words: accretion, accretion disks - instabilities - planetary systems - planetary systems: formation - stars: formation

\section{INTRODUCTION}

The first direct images of extrasolar gas giants present planetary scientists with a new mandate: construct massive planets on extremely wide orbits. Before 2008 November, when the existence of planets surrounding Fomalhaut (Kalas et al. 2008) and HR 8799 (Marois et al. 2008) was revealed, theorists had reached a near-consensus that the core accretion mechanism could explain nearly all observed properties of planets discovered by radial velocities. Simple core accretion-based models reproduce the planet-metallicity correlation (Fischer \& Valenti 2005) and the planet-silicon correlation (Robinson et al. 2006), and the rarity of giant planets orbiting $\mathrm{M}$ dwarfs (Laughlin et al. 2004; Johnson et al. 2007). Unfortunately, with the exception of Ida \& Lin (2004), these models treat only planets forming near the ice line and ignore planet formation beyond $\sim 5 \mathrm{AU}$-for the simple reason that the vast majority of known radial-velocity planets orbit within $3 \mathrm{AU}$ of their host stars. Estimates of the planet occurrence rate beyond 5 AU (e.g., Cumming et al. 2008) rely on extrapolation of the statistics from shorter-period orbits.

One can immediately foresee problems applying the core accretion model - in which a massive solid core destabilizes the surrounding disk gas to accrete an atmosphere-to Fomalhaut b, located 119 AU from its host star, and HR 8799 b, c, and d, with projected stellar separations of 24,38 , and 68 AU. All of these new planets are unequivocally massive and gaseous: the smallest is Fomalhaut b with $M<3 M_{\text {Jup }}$. Even forming the small and solid-rich Uranus and Neptune (14.2 and 17.5 $M_{\oplus}$, respectively) in situ is difficult: Levison \& Stewart (2001) show that the planets cannot form in their current orbits at 19 and

\footnotetext{
4 Formerly Sarah E. Robinson.
}

30 AU because the accretion timescale is far longer than the planetesimal excitation timescale. If accretion timescale prohibits medium-mass planet formation at $19 \mathrm{AU}$, the formation of a gas giant at $>100 \mathrm{AU}$ by core accretion hardly seems possible.

There are a few ways to increase the efficiency of core accretion. Perhaps the protoplanetary disks surrounding HR 8799 and Fomalhaut held far higher planetesimal masses than the solar nebula, and were thus better able to form solid cores at large radii. Since both stars host massive, bright debris belts (Kalas et al. 2005; Chen et al. 2009), this hypothesis requires investigation. It's also possible that these relatively massive stars $\left(\sim 1.5 M_{\odot}, \sim 2.0 M_{\odot}\right.$, respectively) decreased the protoplanet orbital timescale in the outer disk enough for core accretion to proceed. Perhaps it is not a coincidence that the only directly imaged extrasolar planets orbit A stars. In this work, we use model A-, G-, and M-star disks to test the possibility that core accretion at large radii could work for HR 8799 and Fomalhaut where it fails for lower-mass stars.

What are the other possible formation mechanisms for gas giants on wide orbits? For truly massive planets - and in the case of HR 8799, the best-fit masses of $7 M_{\text {Jup }}, 10 M_{\text {Jup }}$, and $10 M_{\text {Jup }}$ are near the $\mathrm{Y}$ dwarf minimum mass (Zuckerman \& Song 2009) — the simplest possibility is fragmentation of the collapsing protostellar core, analogous to binary star formation (e.g., Cha \& Whitworth 2003). However, several lines of observational evidence show that HR 8799 and Fomalhaut are not simply binary or multiple stars with high mass ratios.

1. Resolved images show that both stars host debris disks that are confined by the planet(s), indicating that the planets and debris disks are coplanar relics of protostellar disks (Kalas et al. 2005; Chen et al. 2009; Su et al. 2009). 
2. Spectroastrometric measurements of the spin-orbit alignment of Fomalhaut show that the star's rotation axis is perpendicular to the debris disk and planet orbit, further indicating that Fomalhaut $\mathrm{b}$ formed in a disk (Le Bouquin et al. 2009). The rotational velocity of HR 8799 indicates that it, too, is nearly pole-on and perpendicular to the planet orbits (Reidemeister et al. 2009).

3. The three companions to HR 8799 orbit the central star, rather than forming a double-double system consistent with cloud core fragmentation (Clark \& Bonnell 2005).

As molecular cloud core fragmentation is unlikely, we will treat the companions to HR 8799 and Fomalhaut as bona fide planets (whether they burn deuterium or not) and search for formation mechanisms that take place in protoplanetary disks. The two remaining possibilities are planet-planet scattering, in which planets form in the inner disk and are ejected to wide orbits by a yet-unseen inner planet, and gravitational instability, where the protostellar disk fragments into bound clumps that eventually contract to near Jupiter's radius. Here, too, we run into problems. Planet scattering tends to create either unstable systems or extremely eccentric orbits (Raymond et al. 2008; Veras et al. 2009), whereas the orbits of HR 8799 b, c, and d and Fomalhaut $b$ appear to be nearly circular. There is considerable debate in the literature over whether disk fragments formed in gravitational instabilities survive to become protoplanets or are destroyed by accretion onto the star or background shear flows (Durisen et al. 2007; Vorobyov \& Basu 2006); furthermore, a previous investigation of planet formation on wide orbits by Boss (2006) noted that spiral instabilities may wind too tightly at large radii for the disk to fragment at all. Nonetheless, two points in favor of gravitational instability are that spiral waveswhether they fragment the disk or not-develop most easily in the outer parts of disks, and that when fragments do form they are often near $10 M_{\text {Jup }}$ (Boley 2009; Stamatellos \& Whitworth 2009). Gravitational instability is therefore perfectly positioned to create massive planets with extremely long-period orbits.

In this paper, we present numerical experiments that test the viability of core accretion, planet-planet scattering and gravitational instability. The paper is organized as follows. In Section 2, we describe the disk models that form the foundation of our numerical experiments. In Section 3, we use a simple core-accretion simulation to show that massive core growth on ultra-wide orbits is not possible. We present $\mathrm{N}$-body simulations assessing the likelihood of scattered planets landing in stable, wide orbits in Section 4. The disk stability analysis in Section 5 confirms that our maximum-mass model disks can become unstable to global two-armed spiral modes. Finally, we present our conclusions, along with a proposed observational experiment to confirm that gravitational instability is the dominant mode of planet formation on wide orbits, in Section 6.

\section{DISK MODEL}

We begin by describing the simple disk model that forms the basis for our three numerical experiments. In order to facilitate comparisons of planet formation efficiency among different types of stars, we have deliberately chosen a simple, passively illuminated disk whose temperature profile depends only on the temperature and luminosity of the host protostar.

We use the method of Chiang \& Goldreich (1997) to construct flared protostellar disks that are in radiative equilibrium with their host protostars. In the Chiang \& Goldreich models, dust in a superheated surface layer reprocesses the incident stellar radiation, re-radiating $\sim 1 / 2$ of the absorbed flux onto the disk interior at infrared wavelengths. The midplane temperature of a disk optically thick to radiation from the surface layer is

$$
T_{i} \approx\left(\frac{\alpha}{4}\right)^{1 / 4}\left(\frac{R_{*}}{a}\right)^{1 / 2} T_{*} .
$$

In Equation (1), $a$ is the distance from the star, $R_{*}$ is the protostar radius, $T_{*}$ is the effective temperature, and $\alpha$ is the disk flaring angle:

$$
\alpha=\frac{0.4 R_{*}}{a}+a \frac{d}{d a}\left(\frac{H}{a}\right) .
$$

The first term in Equation (2) can be neglected in the limit $a \gg R_{*}$.

The visible photosphere height is given by

$$
\frac{H}{a}=\frac{H}{h}\left(\frac{T_{*}}{T_{c}}\right)^{4 / 7}\left(\frac{a}{R_{*}}\right)^{2 / 7},
$$

where $h$ is the pressure scale height. The quantity $T_{c}$ defines the escape temperature of the disk gas from the stellar surface:

$$
T_{c}=\frac{G M_{*} \mu}{k R_{*}} .
$$

In Equation (4), $M_{*}$ is the star mass, $\mu$ is the mean molecular weight of the nebula gas in grams, and $k$ is Boltzmann's constant. We take $H / h=4$ as a constant among all model disks. Best-fit spectral energy distributions (SEDs) of protostellar disks have values $H / h$ between 2 and 5 (Chiang et al. 2001).

We test the planet formation efficiency in disks surrounding the types of stars known to host planets (spectral types A-M) by defining three representative protostars: an A star of mass 1.5 $M_{\odot}$, a G star of mass $1.0 M_{\odot}$, and an $\mathrm{M}$ star of mass $0.5 M_{\odot}$. The remaining inputs to Equations (1)-(4), the star radius, and effective temperature, are taken from published pre-main-sequence evolutionary tracks (D'Antona \& Mazzitelli 1994). We choose a stellar age of $10^{5}$ years as the onset of planet formation. Our stars are older than the $100-\mathrm{km}$ planetesimal formation timescale (Barnes et al. 2009), yet still young enough to harbor massive disks, which improves the chances of planet formation. Figure 1 (top panel) shows the midplane temperature as a function of radius for each protostar mass.

We must now calculate the mass available for planet formation. The Chiang \& Goldreich (1997) temperature profile is independent of the disk surface density as long as the disk is optically thick. We therefore use the constraint of local gravitational stability to set the surface density profile. The Toomre $Q$ parameter,

$$
Q=\frac{c_{s} \kappa}{\pi G \Sigma},
$$

measures the stability of the disk to axisymmetric perturbations. In Equation (5), $\kappa$ is the epicyclic frequency, $c_{s}$ is the midplane sound speed, and $\Sigma$ is the gas surface density. Rings of enhanced surface density, with corresponding rarefactions, will grow exponentially in any part of the disk with $Q<1$. With $c_{s}$ determined by the radiative equilibrium model and the assumption of a nearly Keplerian disk, we can recover the surface density profile from Equations (1)-(5) by specifying a value of $Q$ :

$$
\Sigma=\frac{1}{Q \pi}\left(\frac{M_{*}}{G} \frac{k}{\mu}\right)^{1 / 2}\left(\frac{1}{14} \frac{H}{h}\right)^{1 / 8} T_{*}^{4 / 7} T_{c}^{-1 / 14} R_{*}^{3 / 14} a^{-12 / 7} .
$$



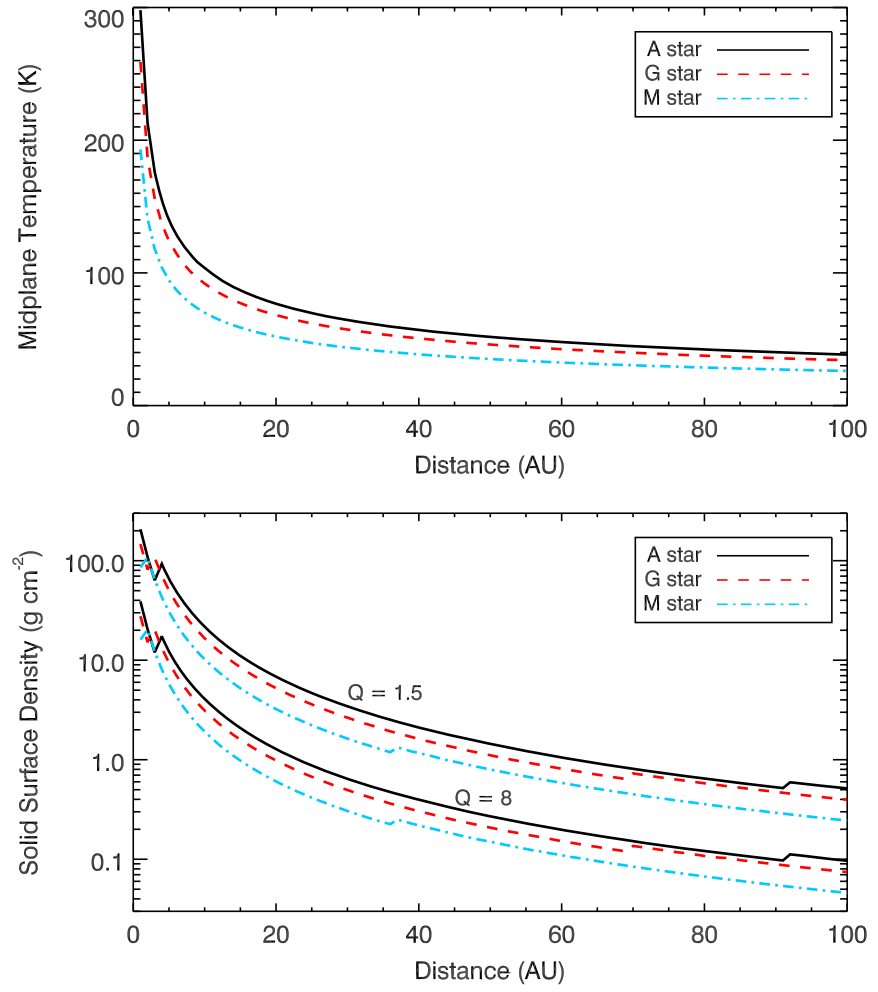

Figure 1. Passive disks in radiative equilibrium with their parent star form the basis of our numerical experiments. Top: midplane temperature as a function of distance from the star for our three model protostars, the $1.5 M_{\odot}$ A star, the $1 M_{\odot} \mathrm{G}$ star, and the $0.5 M_{\odot} \mathrm{M}$ star. Bottom: Solid surface density as a function of distance. The two sets of curves represent the maximum-mass nebulae, with Toomre $Q=1.5$, and the medium-mass nebulae, with $Q=8$. The two discontinuities in the surface density distribution are the water ice line $(<5 \mathrm{AU})$ and the methane condensation front. As long as the nebula is optically thick, midplane temperature is independent of solid surface density.

Since planets on wide orbits are known to be difficult to build (Kenyon \& Bromley 2008; Whitworth \& Stamatellos 2006), we construct our disks with the following question in mind: Are there any circumstances, however optimistic, under which massive planets between 20 and 120 AU can form and survive? We therefore outfit each star with a "maximum-mass nebula," a disk that contains as much mass as it can plausibly hold while maintaining the disklike structure that is critical for core accretion. The maximum-mass nebulae have $Q=1.5$ everywhere in the disk.

Furthermore, since most known planets are not on wide orbits and do not require extremely massive disks to form, we also construct "medium-mass nebulae" with $Q=8$ for each star. The medium-mass nebulae allow us to verify that our calculations of planet formation efficiency are consistent with previous studies of both core accretion and gravitational instability (e.g., Laughlin et al. 2004; Kenyon \& Bromley 2008; Kratter et al. 2008; Stamatellos \& Whitworth 2009).

The final step in constructing our disk models is to account for the viscous accretion that happens before the onset of planet formation, which tends to flatten a strongly centrally peaked surface density profile (Lynden-Bell \& Pringle 1974; DodsonRobinson et al. 2009). We calculate the viscous diffusion timescale, over which surface density decreases by a factor of $\sim 2$, as

$$
t_{\mathrm{diff}}=\frac{a^{2}}{v}=\frac{a^{2}}{\left(\alpha_{\mathrm{visc}} c_{s}^{2} / \Omega\right)^{2}},
$$

where $\alpha_{\text {visc }}$ is the viscous efficiency in the standard $\alpha$-disk model (Shakura \& Syunyaev 1973). Following Dodson-Robinson et al. (2009) and Lyra et al. (2008), we take $\alpha_{\text {visc }}=0.002$. Our final surface density is determined by reducing the fiducial value according to

$$
\Sigma=\Sigma_{0}\left(2^{-10^{5} / t_{\text {diff }}}\right),
$$

where $t_{\text {diff }}$ is given in years. Our method of computing the diffusion timescale includes the assumption that viscous torques provide some angular momentum transport, but stellar radiation dominates the disk energy budget. The effect of diffusion is most profound in the inner disk where $t_{\text {diff }}$ is smallest. The slightly reduced surface density in the inner disk provides some additional gravitational stability to the disk as a whole.

Figure 1 (bottom panel) shows the solid surface density profiles of the maximum-mass and medium-mass nebulae for each star. Each disk has an outer boundary of 100 AU. Gas/ solid ratios are taken from the disk molecular inventory at solar composition of Dodson-Robinson et al. (2009). The maximummass disks hold between $1 / 4$ and $1 / 3$ of the system mass and may undergo global gravitational instabilities that aid planet/ planetesimal formation (e.g., Adams et al. 1989; Rice et al. 2004; Lodato \& Rice 2005), which we examine in Section 5. Such massive disks would be either in the middle or just at the end of their epoch of accretion from the protostellar clump, consistent with our assumed stellar age of $10^{5}$ years. Although we do not examine accretion-triggered instability, it is important to note that the accretion rate onto the disk can be a determining factor for fragmentation at large radii (Vorobyov \& Basu 2006; Boley 2009; Clarke 2009; Rafikov 2009). The medium-mass nebulae hold $<10 \%$ of the total system mass, consistent with disk masses derived from submillimeter observations (Andrews \& Williams 2007).

Having created our disk models, we now test the suitability of each for forming planets.

\section{EXPERIMENT 1: CORE ACCRETION}

Since the astronomical community has reached a nearconsensus that core accretion is the dominant mode of planet formation-explaining such observables as the planetmetallicity correlation (Gonzalez 1998; Fischer \& Valenti 2005), the planet-silicon correlation (Robinson et al. 2006), and the paucity of gas giants orbiting $M$ dwarfs (e.g., Johnson et al. 2007), we begin our investigation of planets on wide orbits with an assessment of the ability to form critical-mass cores at large radii. The quantity of interest is the maximum possible protoplanet core mass as a function of distance from the star. If the core can reach $10 M_{\oplus}$, the canonical critical value for destabilizing the surrounding nebula to accrete a massive atmosphere (Mizuno 1980), while the gas disk is still present, it has a chance of forming a gas giant. We adopt a disk lifetime of $5 \mathrm{Myr}$, the maximum star cluster age with an appreciable disk fraction according to Currie et al. (2009). Here, we are being generous: most disks have far shorter observed lifetimes, but we must account for the possibility that planets on wide orbits are relics of long-lived disks.

We use the feeding zone approximation (Safronov 1969; Lissauer 1993), in which a solitary core accretes planetesimals at the rate

$$
\dot{M}=\pi R^{2} \Sigma \Omega\left(1+\frac{2 G M}{R\langle v\rangle^{2}}\right),
$$

where $M$ is the core mass, $R$ is the core radius, $\Sigma$ is the surface density of planetesimals, $\Omega$ is the Keplerian frequency, and $\langle v\rangle$ 
is the rms planetesimal velocity. Assuming solitary cores tends to increase the maximum planet core mass, since oligarchic growth rates are much slower than those of a single embryo in a swarm of planetesimals. The feeding zone extends 4 Hill radii on either side of the protoplanet (Kary \& Lissauer 1994), where the Hill radius $R_{H}$ demarcates the protoplanet's Roche lobe in the spherical approximation:

$$
R_{H}=a\left(\frac{M}{3 M_{*}}\right)^{1 / 3} .
$$

In Equation (10), $a$ is the planet's semimajor axis and $M_{*}$ is the star mass. We begin the calculation with a $0.1 M_{\oplus}$ seed core and use a fourth-order Runge-Kutta method to integrate Equation (9) for $5 \mathrm{Myr}$, our adopted maximum disk lifetime. We consider only the solid core growth and do not treat gas accretion.

To calculate the gravitational focusing factor (the ratio of the core escape velocity to the mean planetesimal velocity), we apply the simple assumption that $\langle v\rangle=R_{H} \Omega$, so that planetesimals are at the Hill velocity. Accretion is therefore on the threshold between dispersion-dominated, where only two-body forces (protoplanet and planetesimal) are important, and shear-dominated, where the entire star-planet-planetesimal system must be treated (Greenzweig \& Lissauer 1992). Since three-body accretion rates tend to be lower than two-body rates, our core accretion model may provide optimistically high estimates of the core accretion efficiency. However, as we shall see, core accretion cannot form gas giants at large radii even under the extremely favorable conditions we have constructed here.

Figure 2 shows the maximum planet core mass as a function of distance from the star. In the medium-mass A and G star nebulae, giant planet formation is comfortably confined to a narrow belt near $10 \mathrm{AU}$ : there is no chance of forming a super-Jovian planet beyond $20 \mathrm{AU}$. The maximum core mass barely reaches the critical $10 M_{\oplus}$ in the G star disk, confirming that giant planet formation by core accretion is a threshold phenomenon. A slight downward adjustment in disk mass, metallicity, or lifetime would be enough to prevent any giant planets from formingand indeed, the estimated planet occurrence rate between 0 and 5 AU is only $10 \%$ for solar-mass stars (Cumming et al. 2008). The medium-mass $\mathrm{M}$ star disk does not form any giant planets at all, consistent with the observed paucity of gas giants orbiting M dwarfs (Johnson et al. 2007).

We note that critical core mass is not constant throughout the disk, but instead depends on the isolation mass of planetesimals in the feeding zone. In the outer disk, where the isolation mass can reach $100 M_{\oplus}$ or more, the solid accretion rate remains high throughout planet formation and the kinetic energy deposited by planetesimals inhibits the protoplanet atmosphere's contraction. By using $10 \quad M_{\oplus}$ as the critical core mass throughout our experiments, we are overestimating the chances of giant planet formation in the outer disk: simulations of planet formation in the trans-Saturnian solar nebula show that critical mass can exceed $40 M_{\oplus}$ (Dodson-Robinson et al. 2008; DodsonRobinson \& Bodenheimer 2009).

Now, being extremely generous not only with the feeding zone setup and disk lifetime, we investigate the core formation efficiency in the maximum-mass nebulae. Since core accretion requires a stable, quiescent disk, we cannot possibly load the disk with more planetesimal mass than contained in the $Q=1.5$ nebulae (except through second-order effects such as high metallicity). If giant planet cores cannot reach critical mass in our maximum-mass nebulae, they have no chance to
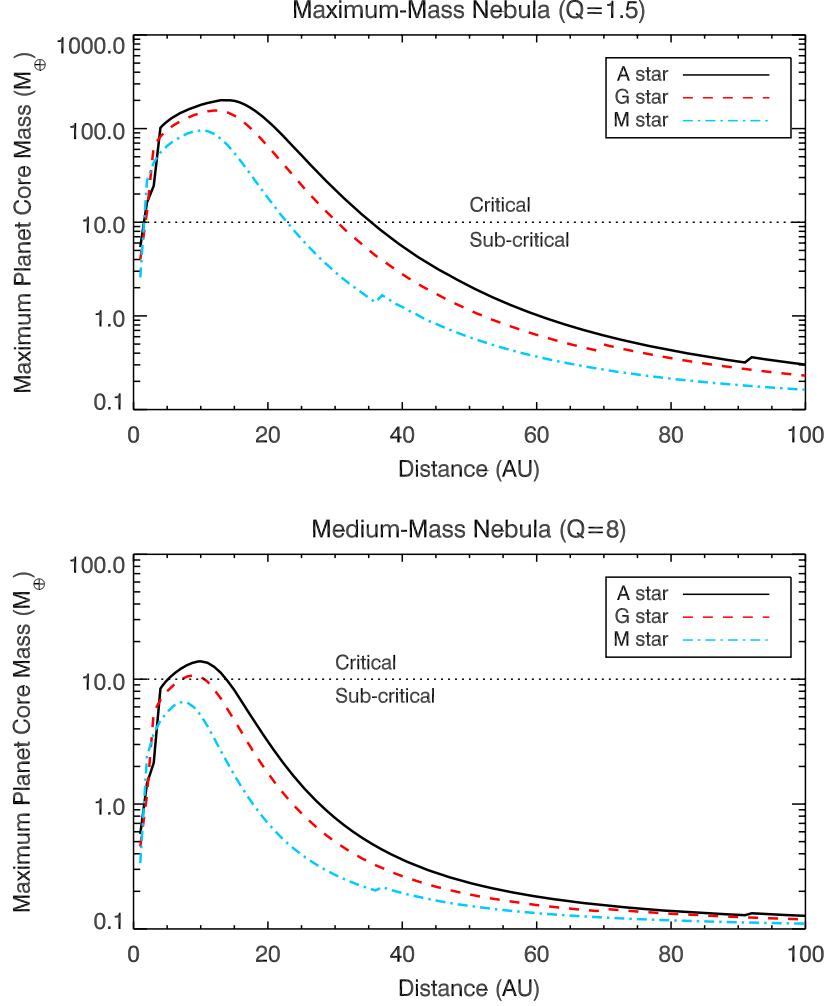

Figure 2. Even our model A star hosting its maximum-mass nebula cannot form gas giant planets by core accretion at distances larger than 35 AU. Top: maximum planet core mass as a function of distance in the maximum-mass nebulae. Assuming a critical core mass of $10 M_{\oplus}$, the giant planet-forming region is restricted to $a<35 \mathrm{AU}$ in the A star disk, $a<31 \mathrm{AU}$ in the $\mathrm{G}$ star disk, and $a<23 \mathrm{AU}$ in the M star disk. Bottom: In the more typical mediummass nebulae, giant planet formation is restricted to a narrow region centered at $\sim 10 \mathrm{AU}$ in the model $\mathrm{A}$ and $\mathrm{G}$ star disks. The $\mathrm{M}$ star disk is not capable of forming any gas giants by core accretion.

do so in any physically realistic disk. Figure 2 shows that the maximum radius where core accretion is effective is $35 \mathrm{AU}$ in the A star disk, 31 AU in the G star disk, and 23 AU in the M star disk. Core accretion might be responsible for HR $8799 \mathrm{~d}$, on a 23 AU orbit (Marois et al. 2008; Fabrycky \& Murray-Clay 2008), but the other direct imaging discoveries HR $8799 \mathrm{~b}$ and $\mathrm{c}$ and Fomalhaut $\mathrm{b}$ are well outside the core accretion planetforming zone.

Is it possible that a super-Jupiter could form on a $\sim 35 \mathrm{AU}$ orbit and migrate outward to 50 AU or greater? Perhaps: there are two robust outward migration mechanisms, planetesimal scattering and Type III migration. In addition, Morbidelli \& Crida (2007) propose that two giant planets in a 2:3 mean motion resonance that open overlapping gaps in the disk may halt or reverse the normally inward Type II migration process.

Hahn \& Malhotra (1999), followed by Gomes et al. (2004) and Tsiganis et al. (2005), found that a 30-50 $M_{\oplus}$ planetesimal disk could expand Neptune's orbit from 23 AU to 30 AU. However, a planet must interact with at least its own mass in planetesimals to change its semimajor axis appreciably. Even our maximummass A star nebula contains $<2 M_{\text {Jup }}$ of planetesimals between 35 and 100 AU. Since HR 8799 b and c are each at least $7 M_{\mathrm{Jup}}$, we can rule out core accretion followed by planetesimal-driven migration.

Type III migration, which is driven by torques at the planet's corotation resonance and may proceed inward or outward, is the least understood type of large-scale planet motion. However, Pepliński et al. (2008) show that outward Type III migration (1) 
requires a sharp inner disk edge located near the planet and (2) always reverses direction and becomes inward migration after the planet's semimajor axis has approximately doubled. Based on their work, we do not consider Type III migration from the inner disk a viable explanation for the origins of HR $8799 \mathrm{~b}$ and Fomalhaut b.

Our first numerical experiment demonstrates that even under the most favorable conditions possible-massive, long-lived disk; low-velocity planetesimals; a seed core of $0.1 M_{\oplus}$ to start the accretion-gas giants cannot form by in situ core accretion beyond $35 \mathrm{AU}$. For lower-mass $\mathrm{G}$ and $\mathrm{M}$ stars, the core accretion scenario is even more unfavorable. The work of Peplinski et al. (2008) indicates that outward migration from the inner disk is also implausible. The next two experiments investigate other ways of placing super-Jovian planets on extremely wide orbits.

\section{EXPERIMENT 2: SCATTERING}

Next, we consider the possibility that planetary systems such as HR 8799 may in fact have formed via core accretion-but as a system that was initially more compact than currently observed and containing at least one additional massive planet. (Here, we use the term "planet" despite the significant uncertainties in mass and formation mechanism.) The unseen, massive planet scatters the discovered planets, forming in the inner nebula according to the canonical core accretion theory, out to their current wide orbits. Since HR 8799 is the only multi-planet system that includes planets on ultra-wide orbits, we use it as a test case for this experiment.

Our basic approach is to perform many $\mathrm{N}$-body integrations of systems with a $1.5 M_{\odot}$ A star, to match the mass of HR 8799, and four planets. Our goal is to determine if planet scattering can lead to systems qualitatively similar to the HR 8799 system. Three of the planets correspond to the discovered objects, with masses of $7 M_{\text {Jup }}, 7 M_{\text {Jup }}$, and $5 M_{\text {Jup }}$. These masses are intentionally chosen to be near the lower limit of current mass estimates (Marois et al. 2008), as previous studies have shown that systems containing lower planet masses are less likely to be rapidly disrupted by strong planet-planet scattering (Fabrycky \& Murray-Clay 2008; Goździewski \& Migaszewski 2009). The putative fourth companion would need to have a mass equal to or larger than the other planets so that it can effectively scatter the other planets outward (Ford \& Rasio 2008). Since the total mass in planets is already quite large compared to other known planetary systems (Butler et al. 2006), we set the mass of the putative fourth body to be $7 M_{\mathrm{Jup}}$. We integrate each initial configuration of planets for $200 \mathrm{Myr}$, using the Mercury hybrid symplectic integrator (Chambers 1999).

The initial semimajor axis for the unseen inner planet is drawn from a uniform distribution between 3 and 7 AU. The lower limit is chosen in order to place the innermost planet as close to the star as it could plausibly have formed via core accretion. As shown in Figure 2 (black curve), for A stars there is a rapid rise in the maximum core mass near $3 \mathrm{AU}$ in both the medium- and maximum-mass nebulae. The upper limit for the initial semimajor axis of the inner planet is based on the need for the outer planets to be close enough to the central star for core accretion to remain a viable formation mechanism even when the planets are placed several mutual Hill radii apart (see below).

We assign initial semimajor axes for the three outer planets such that the difference in the semimajor axis of each each pair of neighboring planets is $a_{i+1}-a_{i}=K \times r_{H, i, i+1}$, where $K$ is a constant of order unity and $r_{H, i, i+1}$ is the mutual Hill radius of the $i$ th and $i+1$ th planet (Chatterjee et al. 2008):

$$
r_{H, i, i+1}=\left(\frac{M_{i}+M_{i+1}}{3 M_{*}}\right)^{1 / 3} \frac{a_{i}+a_{i+1}}{2} .
$$

The choice of $K$ affects the spacing of the planets and thus the timescale until the first close encounter. Based on similar scattering experiments (but with three less massive planets), we expect that a small choice for $K$ (e.g., $\sim 2-3.5$ ) is likely to result in a very rapid first scattering event, which would cast doubt on the plausibility on the initial conditions. A somewhat larger choice of $K \sim 4$ allows the system to evolve for hundreds or thousands of orbital periods before the first close encounter. Thus, the scattering is triggered by dynamical instability in a chaotic system rather than the choice of initial conditions (Chatterjee et al. 2008). In this case, we cannot incrase $K$ much further, as the initial semi-major axis of the outermost planet becomes prohibitively large in the context of formation via core accretion (Figure 2).

Each planet is assigned an initial eccentricity of less than 0.05 , an initial inclination of less than 0.025 radians, and random angles for the initial pericenter direction, initial longitude of ascending node and initial mean anomaly. We perform two sets of $\simeq 300$ simulations, one with $K=4.0$ and another with $K=4.5$. The sequence of the three observed planets is chosen randomly.

The median initial semimajor axis of the outer-most planet is $33.8 \pm 5.0$ AU for $K=4.0$ and 40.2 \pm 7.0 AU for $K=4.5$. Thus, three of the simulated planets typically start at smaller semimajor axes than the observed separations for all three observed companions. One simulated planet typically has an initial semi-major axis between HR 8799 c and d in the $K=4.0$ simulations, or comparable to the present separation of planet $\mathrm{c}$ in the $K=4.5$ simulations. As shown in Figure 2 (black curve), the maximum core mass cannot reach critical core mass beyond separations of $\sim 35 \mathrm{AU}$. If taken literally, this would suggest that only our simulations with an inner planet initially near 3 AU could result in four giant planets with each separated from its neighbors by at least 4 mutual Hill radii. Nevertheless, we consider initial conditions that include systems with somewhat wider initial spacings, given the large current separations of planets HR8799 b, c, and d. While the initial conditions with $K=4.0$ are more consistent with the modeling in Section 3, most of these simulations result in rapid instabilities (within $\sim 1$ Myr or $\sim 5000$ orbital periods of the outer planet). This motivated the additional simulations with $K=4.5$ which had their first close encounter on a longer timescale of $\sim 24 \mathrm{Myr}$, near the minimum age of the host star (30 Myr; Marois et al. 2008).

For the simulations with $K=4.5, \simeq 32 \%$ of the systems retained exactly three planets at the end of the integrations. With $K=4.0$, the retention rate was far worse: $\leqslant 1 \%$ of the systems retained exactly three planets. In both cases, the systems that retained three planets did not have architectures similar to HR 8799, since one of the planets had migrated inwards and thus only two remained at large separations. Some of these systems did not suffer an orbital instability (so no planets at separations comparable to HR 8799b), while others had planets with final semimajor axes quite different from those of HR 8799 (e.g., outermost planet with a semimajor axis much greater than the other planets). Thus, our simulations suggest that planet scattering alone cannot produce a stable system that looks like HR 8799_although Fomalhaut b, with a solitary planet on a wide orbit, might be possible. 
The above result leads us to consider the possibility that HR 8799 , and future discoveries of multiple, massive planets on wide orbits, might be dynamically unstable. Given the relatively young age of the host star ( $<160$ Myr; Marois et al. 2008), we might be observing the planetary system in a transient phase while planet-planet interactions are still acting to resolve a recent dynamical instability (Goździewski \& Migaszewski 2009). Thus, we analyzed snapshots of each system taken every 200,000 years up to a maximum duration of $160 \mathrm{Myr}$. We include snapshots at times as small as 200,000 years after the beginning of our $N$-body integrations.

We identify those snapshots with three outer planets that have semimjaor axes between 20 and $100 \mathrm{AU}$, qualitatively similar to HR $8799 \mathrm{~b}$, c and d, plus a fourth planet that is closer to the star and would have eluded detection. For the simulations with $K=4.0$ (4.5), we find $\sim 0.01 \%-0.07 \%(\sim 0.01 \%-0.02 \%)$ of snapshots meet this criteria depending on whether we look at the entire $160 \mathrm{Myr}$ or just the first $30 \mathrm{Myr}$ of our simulations. These simulations suggest that if the HR 8799 system is the result of core accretion at smaller separations followed by planet-planet scattering, then we would be observing the system during a very brief transient phase of its evolution. Thus, planet scattering is not a viable formation model if systems such as HR 8799 are found to be common. However, at least for the time being, HR 8799 is the only multi-planet system discovered by direct imaging and likely subject to strong selection effects.

In summary, we cannot conclusively rule out the model of core accretion followed by planet scattering for young systems that might still be dynamically evolving. Our results are consistent with the findings of Goździewski \& Migaszewski (2009), Scharf \& Menou (2009) and Veras et al. (2009), who find dynamical relaxation timescales of only $10 \mathrm{Myr}$ for tightly packed systems. Indeed, Scharf \& Menou report a planet retention rate of $20 \%$ after 10 Myr. Furthermore, Raymond et al. (2009) show that while planetesimals may help retain planets that would otherwise have been ejected, the eccentricity distribution of massive scattered planets remains wide-contrary to the case of HR 8799 where stability requires nearly circular orbits (Lafrenière et al. 2009). Even Fomalhaut b, with a minimum eccentricity of 0.13 (Kalas et al. 2008), cannot have a periapseapoapse swing of more than $15 \mathrm{AU}$ without disrupting its neighboring debris disk. We recommend that observers examine the planet detection rate as a function of stellar age, controlling for the planets' dimming with time. If planet detection rate is found to be independent of stellar age, it would confirm our finding that planet scattering is not the dominant mode of producing detectable planets on wide orbits.

Finally, there is the possibility-not explored here-that large planet masses may allow for significant planet-planet interactions to begin occurring while there are still significant planet-disk interactions (e.g., migration, eccentricity damping, and accretion) that are not included in our model. Unfortunately, detailed models of planet-disk interactions over $\sim 100 \mathrm{Myr}$ would be extremely computationally demanding and are thus left for a future study.

\section{EXPERIMENT 3: GRAVITATIONAL INSTABILITY}

Our planet scattering experiment was not successful at making a stable system with massive planets on wide orbits, and we showed in Section 3 that core accretion plus migration have severe difficulties producing massive gas giants on wide orbits. In this section, we investigate the one remaining possibility, gravitational instability in the disk. Following (1) the breakup of a disk into fragments, (2) the orbital evolution of each fragment, (3) the fragments' late-stage accretion of both planetesimals and gas, and (4) the contraction of a fragment to approximately Jupiter's radius is beyond the capability of any single numerical model. As a first step, we simply verify that our model disks can become gravitationally unstable.

\subsection{Mathematical Method}

We search for exponentially growing spiral modes using the linear stability analysis method of Adams et al. (1989), further developed by Laughlin \& Rozyczka (1996). The method is described extensively in the literature, so we provide only an overview here.

The disk surface density is decomposed into an unperturbed component plus an exponentially growing mode in spiral form:

$$
\Sigma(r, \theta, t)=\Sigma_{0}(r)+\Sigma_{1}(r) \exp [i(\omega t-m \theta)] .
$$

In Equation (12), $\Sigma_{0}$ is the unperturbed surface density, $\Sigma_{1}$ is the perturbed component, $m$ is the number of spiral arms, and $(r, \theta, t)$ are the radial, azimuthal, and time coordinates. The complex eigenfrequency $\omega$ is given by

$$
\omega=m \Omega_{p}-i \gamma,
$$

where $\Omega_{p}$ is the fixed pattern speed and $\gamma$ is the growth rate of the spiral wave. The disk radial velocity, azimuthal velocity and potential $\psi$ due to self-gravity can be modally decomposed in an analogous manner to the surface density. Unperturbed quantities $\Sigma_{0}$ and $c_{s}$ (sound speed) are specified by the disk models described in Section 2. Here, we assume that the disk is vertically isothermal, consistent with the Chiang \& Goldreich (1997) passive disk model adopted in Section 2.

We begin our search for growing modes by calculating the rotation curve-including self-gravity and pressure supportand corresponding epicyclic frequency:

$$
\begin{gathered}
r \Omega^{2}(r)=\frac{G M_{*}}{r^{2}}+\frac{c_{s}^{2}}{\Sigma_{0}} \frac{d \Sigma_{0}}{d r}+\frac{d}{d r}\left(\psi_{0}\right), \\
\kappa^{2}(r)=\frac{1}{r^{3}} \frac{d}{d r}\left[\left(r^{2} \Omega\right)^{2}\right] .
\end{gathered}
$$

The disk self-gravity term, the third term on the right-hand side of Equation (14), is numerically evaluated as

$$
r \Omega_{\mathrm{disk}}^{2}=-\frac{d}{d r} \int_{0}^{2 \pi} d \theta \int_{R_{\mathrm{in}}}^{R_{\mathrm{out}}} \frac{G \Sigma_{0}\left(r^{\prime}\right) r^{\prime} d r^{\prime}}{\left(r^{2}+r^{\prime 2}-2 r r^{\prime} \cos \theta+\eta^{2}\right)^{1 / 2}}
$$

where the softening parameter $\eta=0.1$ allows the azimuthal integral, which diverges at the disk edges, to be evaluated.

The next step is to calculate the potential due to self-gravity of the growing spiral perturbation:

$$
\psi_{1}=-G \int_{R_{\text {in }}}^{R_{\text {out }}} \Sigma_{1}\left(r^{\prime}\right) r^{\prime} d r^{\prime} \int_{0}^{2 \pi} \frac{d \theta}{\sqrt{r^{2}+r^{\prime 2}-2 r r^{\prime} \cos \theta}} .
$$

We define the perturbed component of the disk enthalpy, which works against the growth of the spiral mode, as

$$
h_{1}=c_{s}^{2} \frac{\Sigma_{1}}{\Sigma_{0}}
$$

The linear stability analysis method is built on the approximation that density waves propagate only radially and azimuthally. 
Table 1

Fastest-growing Modes in Maximum-mass Disks

\begin{tabular}{|c|c|c|c|c|c|}
\hline Star Type & $\Omega$ At Disk Edge $\left(s^{-1}\right)$ & Pattern Speed ${ }^{\mathrm{a}}$ & Growth Rate & Corotation (AU) & Position in Figure 3 \\
\hline \multirow[t]{3}{*}{$\mathrm{A}$} & $2.93 \times 10^{-10}$ & 15.5 & 3.2 & 15.2 & Not pictured \\
\hline & & 2.1 & 1.1 & 59.4 & Lower left \\
\hline & & 5.1 & 0.5 & 32.3 & Upper left \\
\hline \multirow[t]{3}{*}{$\bar{G}$} & $2.44 \times 10^{-10}$ & 3.9 & 7.1 & 38.8 & Upper middle \\
\hline & & 3.2 & 2.9 & 44.4 & Not pictured \\
\hline & & 4.9 & 2.5 & 51.6 & Lower middle \\
\hline \multirow[t]{2}{*}{$\mathrm{M}$} & $1.79 \times 10^{-10}$ & 5.4 & 4.2 & 30.2 & Upper right \\
\hline & & 2.4 & 2.5 & 53.7 & Lower right \\
\hline
\end{tabular}

Note. ${ }^{a}$ Pattern speed and growth rate are given in units of the orbital angular speed $\Omega$ at the outer disk edge.

Such a formulation requires that we treat the disk as a twodimensional surface, so we cannot use volume density in our calculations. Therefore, although we have introduced enthalpy into the calculation to treat adiabatic density perturbations, we use the isothermal sound speed, which does not depend on density, in Equations (14) and (17).

Finally, we combine the linearized equations of motion (continuity, radial force and azimuthal force; written out in full in Adams et al. 1989) into a single homogeneous integrodifferential equation in terms of $h_{1}$ and $\psi_{1}$ :

$$
\frac{d^{2}}{d r^{2}}\left(h_{1}+\psi_{1}\right)+A \frac{d}{d r}\left(h_{1}+\psi_{1}\right)+B\left(h_{1}+\psi_{1}\right)+C h_{1}=0,
$$

with the coefficients $A, B, C$ and $v$ defined as

$$
\begin{aligned}
A & =\frac{d}{d r} \log \left[\frac{\Sigma_{0} r}{\kappa^{2}\left(1-v^{2}\right)}\right], \\
B & =-\frac{m^{2}}{r^{2}}-\frac{4 m}{r^{2}} \frac{\Omega}{\kappa} \frac{r}{\left(1-v^{2}\right)} \frac{d v}{d r}+\frac{2 m}{r v} \frac{\Omega}{\kappa} \frac{d}{d r} \log \left(\frac{\kappa^{2}}{\Omega \Sigma_{0}}\right), \\
C & =-\frac{\kappa^{2}\left(1-v^{2}\right)}{c_{s}^{2}} \\
\nu & \equiv \frac{\omega-m \Omega}{\kappa} .
\end{aligned}
$$

For the full derivation of the governing equation, see Adams et al. (1989).

The goal of linear stability analysis is to find combinations of $m, \Omega_{p}$, and $\gamma$ that satisfy Equation (18). Such combinations represent exponentially growing spiral modes. Since we do not a priori know the perturbed surface density $\Sigma_{1}$ associated with any mode, we discretize the disk and write the governing equation in matrix form:

$$
\mathcal{M}_{j k} \Sigma_{1_{k}}=0 \text {. }
$$

In Equation (20), $\Sigma_{1_{k}}$ is the perturbed surface density at position $k$ of a discrete, linear grid. The matrix $\mathcal{M}_{j k}$ acts on the perturbed surface density to evaluate the left-hand side of Equation (18). Since Equation (18) is homogeneous, it has a solution if the complex eigenfrequency $\omega$ (Equation (13)) is an eigenvalue of $\mathcal{M}_{j k}$, such that

$$
\operatorname{det}\left|\mathcal{M}_{j k}\right|=0 .
$$

To construct $\mathcal{M}_{j k}$, we identify the rows with variable $r$ in Equation (16) and the columns with variable $r^{\prime} . \mathcal{M}_{j k}$ must encode a method of integrating Poisson's equation (Equation (16)), which is singular on the diagonal, to calculate $\psi_{1}$. For the diagonal elements of $\mathcal{M}_{j k}$, we use open-interval Romberg integration
(Press et al. 1992) to evaluate the azimuthal integral. We incorporate coefficients of the extended trapezoidal rule to perform the integration over $r^{\prime}$. The top and bottom rows of matrix $\mathcal{M}_{j k}$ encode the boundary conditions describing the flow of gas at the inner and outer disk edges. We follow Laughlin \& Korchagin (1996) in requiring the radial component of the velocity to vanish at both boundaries. The effect of these boundary conditions is to forbid the disk from expanding inward or outward, which results in lossless reflection of density waves at the disk boundaries. The rigid boundary approximation and the value of the softening parameter $\eta$ in Equation (15) combine to artificially affect stability at the disk edges (Adams et al. 1989).

Finally, we need a way of honing in on eigenvalues that provide solutions to Equation (18). We restrict our analysis to two-armed spirals and do not consider higher-order modes. Viable pattern speeds have their corotation resonances inside the disk, so we check values of $\Omega_{p}$ that lie along the rotation curve (Equation (14)). We desire modes that grow within a few to a few hundred orbital periods, so they can substantially modify the structure of the disk before accretion or stellar heating stabilizes it: the growth rate is therefore constrained by $0.01 \Omega_{p} \lesssim \gamma \lesssim \Omega_{p}$. We use the Newton-Raphson method to identify the valid eigenmodes beginning with gridded guesses for $\Omega_{p}$ and $\gamma$. Once we have found a solution to Equation (21), we set the first component of the perturbed surface density vector to unity and use least-squares analysis to solve the overconstrained system resulting from Equation (20).

\subsection{Results}

Our linear stability analysis reveals that the maximum-mass nebulae do indeed undergo global gravitationally instabilities. In all the maximum-mass disks, regardless of host star mass, we found multiple $m=2$ growing modes with corotation resonances inside the disk. We list the fastest-growing modes uncovered by the Newton-Raphson algorithm-those with growth rates greater than $10 \%$ of the orbital speed at the outer disk edge-in Table 1. For each mode the maximum-amplitude perturbations straddle the corotation resonance and are contained within the inner and outer Lindblad resonances, which occur at $\Omega_{p}=\Omega \mp \kappa / 2$. Figure 3 shows six of the modes listed in Table 1. Units on the color scale are arbitrary as the modes grow exponentially. In our simple formulation, where the disk characteristics are completely determined by the stellar radius and temperature, the viability of spiral modes does not appear to depend on the mass of the star: any system with a high disk/star mass ratio can become unstable.

Since our maximum-mass disks are both thin and massive relative to their host stars, containing between 1/4 (A star) 

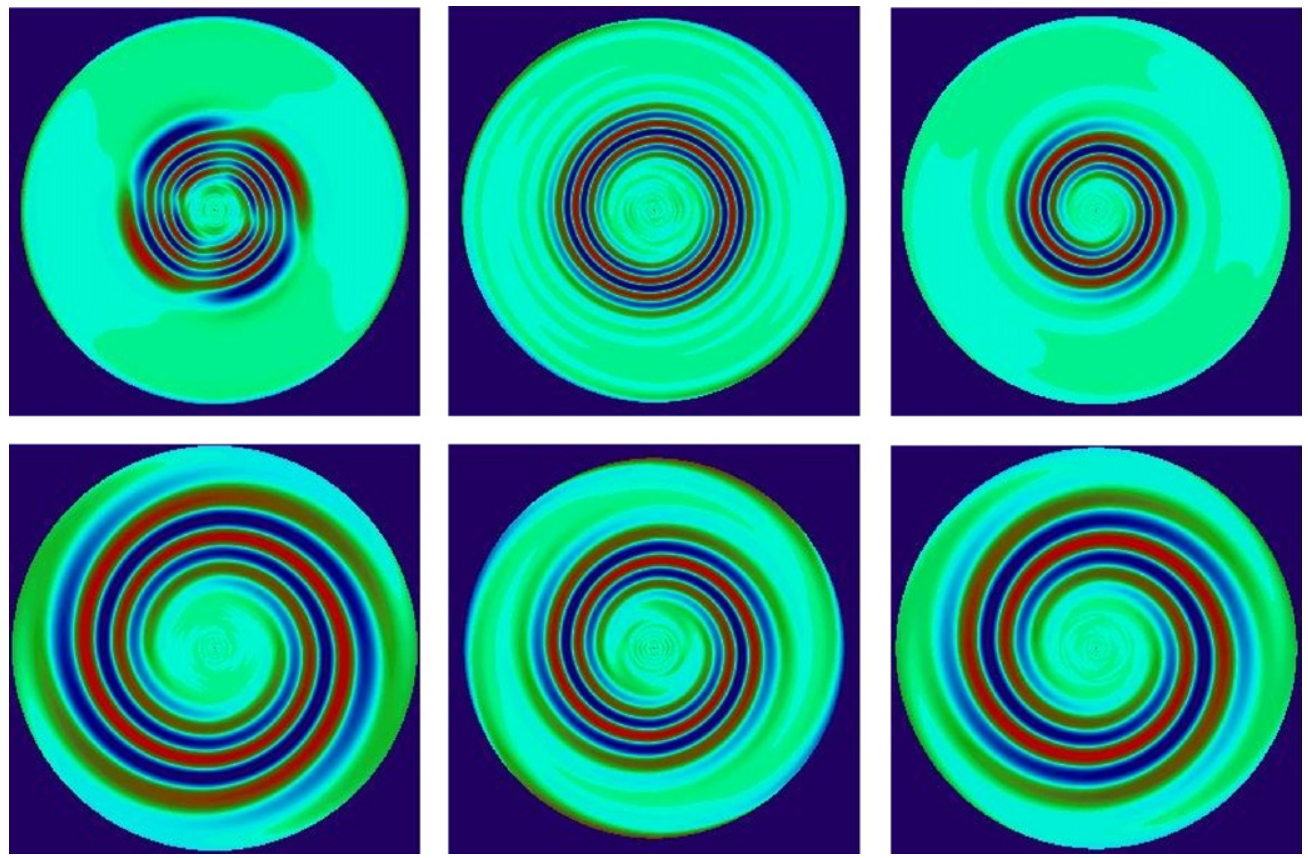

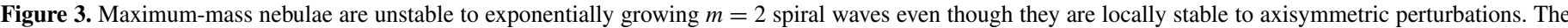

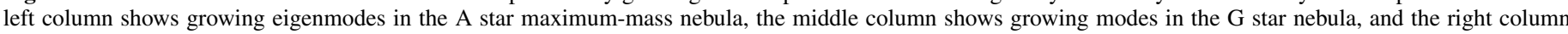

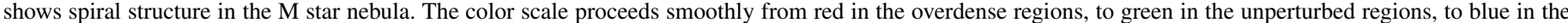

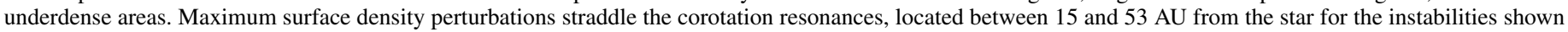
here. Our numerical experiments demonstrate that gravitational instability is the only viable planet formation mechanism for planets on wide ( $a \gtrsim 35$ AU) orbits.

and $1 / 3$ (M star) of the total system mass, the fact that they become unstable is not at all surprising. Numerous previous investigations, including Adams et al. (1989), Laughlin et al. (1998), Lodato \& Rice (2005), Kratter et al. (2008), Cai et al. 2008, and Stamatellos \& Whitworth (2009), have verified that cold disks with more than $10 \%$ of the mass of their stars are subject to long-wavelength, global gravitational instabilities.

Again unsurprisingly, we were not able to find growing modes in our medium-mass nebulae within the limitations of a gridbased search for modes using the Newton-Raphson scheme. If planets on wide orbits form by gravitational instability, they can do so only in massive disks. They must be relics of the early (Class 0-Class 1) stages of protostar evolution, where massive disks are still present.

At long last one of our experiments has revealed that there is a chance of forming the companions to HR 8799 and Fomalhaut, and placing them on stable orbits, by a known mechanism. However, there is still a lot of work to be done to confirm that these planets are products of gravitational instability. Our experiments have merely verified that our maximum-mass disks can become unstable. We do not yet know what happens to the resulting spiral modes. We must rely on the work of previous investigators, whose simulations have shown spiral arms in massive disks can fragmenting into self-gravitating clumps of several Jupiter masses (e.g., Boss 2000; Pickett et al. 2003; Mayer et al. 2004; Boley 2009). Even more importantly, simulations by Mayer et al. (2004) and Boss (2005) indicate that the protoplanetary clumps can survive without migrating into the star.

We caution that the viability of disk instability as a planet formation mechanism has not been fully established. The fragment survival issue requires further investigation, as Mejía et al. (2005) show that clumps may be sheared apart within an orbit or less. Pickett \& Durisen (2007) caution that clump longevity in numerical simulations strongly depends on artificial viscosity, which is implemented differently in smoothed particle hydrodynamics and grid-based calculations. Boley et al. (2007) and Cai et al. (2009) improve upon the radiative transfer treatment of Boss (2007), who found rapid disk cooling and fragmentation, and recover a cooling rate too slow for any fragmentation to occur over several outer disk rotations. The emerging consensus seems to be that while fragmentation cannot occur in the inner disk, $a \lesssim 20-40$ AU (Rafikov 2005; Boley \& Durisen 2008; Stamatellos \& Whitworth 2008; Cai et al. 2009; Forgan et al. 2009), it does occur in extended disks outside of a few tens of AU (Boley 2009; Stamatellos \& Whitworth 2009) provided that the disks are massive. The analytical work of Clarke (2009) demonstrates that disks accreting from the protostellar core inevitably fragment upon reaching a radial extent of $70 \mathrm{AU}$.

At this point, we have only circumstantial evidence that massive planets on wide orbits, beyond $35 \mathrm{AU}$, form by gravitational instability. In this work, disk instability emerges as the only possible theory from a "survival of the fittest" experiment, but it is possible that some fundamental piece of physics is missing from our understanding of planet formation. Nevertheless, unless the wide-orbit planetary systems we have observed are dynamically unstable - unlikely given their apparently near-circular orbitsit seems like there is no other possibility. Core accretion at large stellar separations unequivocally does not work. Our numerical experiments demonstrate that gravitational instability is the only viable planet formation mechanism for planets on wide ( $a \gtrsim 35$ AU) orbits. We predict that HR 8799 b, c, and d, Fomalhaut $b$, and similar planets that may yet be discovered are the result of gravitational instabilities in protostellar disks.

Boley (2009) argues for two modes of planet formation, with core accretion dominating near the star and disk instability forming a population of gas giants at large radii. To Boley's 
conclusion that super-Jupiters on wide orbits should be a significant, or even dominant, component of the gas giant population of low-metallicity stars-in whose disks core accretion is inefficient-we add that the same is true of low-mass stars. Figure 2 demonstrates that gas giant formation by core accretion is all but impossible for $\mathrm{M}$ dwarfs. Core accretion has a chance only in M-star disks with extremely high mass and/or metallicity, but high-mass disks are likely to fragment and destroy the quiescent feeding zones necessary for steady growth by core accretion.

Clarke (2009) argues that companions formed by disk fragmentation typically have at least $10 \%$ of the primary star mass. The minimum-mass disk-formed companion to a $0.1 M_{\odot}$ star would therefore be 10 Jupiter masses and would be classified as a planet rather than a brown dwarf. We predict that the occurrence ratio of long-period to short-period gas giants should be highest for $M d$ warfs, reflecting both the inefficiency of short-period planet formation by core accretion and the planetary-scale minimum mass of wide-orbit fragments formed by disk instability.

Having finally identified a plausible formation mechanism for massive planets in wide orbits, we conclude our article with a review of our findings and recommendations for observers on how to confirm the gravitational instability hypothesis.

\section{CONCLUSIONS AND TESTABLE PREDICTIONS}

We have investigated the likelihood of forming massive planets on wide orbits by three methods: nearly in situ core accretion, scattering by an unseen companion, and gravitational instability. Highlighting the theoretical difficulties presented by the companions to Fomalhaut and HR 8799, the prospects for forming such planets are generally poor. We can certainly rule out core accretion, as it is hard to imagine a way around the fact that long dynamical times destroy the chances of building critical-mass cores. So far, numerical simulations of Type III migration do not indicate that there is any possibility of moving a planet all the way from $35 \mathrm{AU}$ - the largest possible stellar separation at which gas giants can form by core accretion-to $>60$ AU (Pepliński et al. 2008).

Planet-planet scattering can at least move a planet into a wide orbit; the difficulty is keeping it there. Our $N$-body simulations (Section 4) demonstrate that once scattered, planets tend to leave the system. Planetesimals-not included in our calculationscan stabilize the orbits of scattered planets through dynamical friction. However, a planet needs to interact with of order its own mass in planetesimals to circularize its orbit. Even our maximum-mass A star nebula, with total mass $0.51 M_{\odot}$ within $100 \mathrm{AU}$, does not contain 7-10 $M_{\text {Jup }}$ of planetesimals beyond 35 AU. If HR 8799 is an unstable system, planetplanet scattering may be responsible for its architecture, but the shepherded debris disk surrounding Fomalhaut (Kalas et al. 2005) makes its present dynamical instability unlikely.

The one planet formation mechanism that can succeed at creating massive gas giants on wide, near-circular orbits is gravitational instability. In Section 5, we demonstrated that the same maximum-mass disk models which could not form planets on wide orbits by core accretion do undergo global spiral instability. We predict that massive gas giants on wide orbits are the products of protostellar disk fragmentation. This prediction, however, comes with the caveat that the detailed physics of the disk instability process must be further analyzed and the clump shearing and disk cooling time problems resolved (Mejía et al. 2005; Cai et al. 2006; Pickett \& Durisen 2007; Rafikov 2009).
A simple observational test would resolve the question of how massive planets on wide orbits form. So far 11 planets or Y dwarfs orbiting main-sequence stars have been discovered by direct imaging. Once the number of direct imaging discoveries reaches $\sim 30$, we can begin to look for simple statistical patterns in the properties of planet hosts. We propose dividing the stars surveyed by direct imaging into young and old age bins, with the dividing age of order $\sim 100 \mathrm{Myr}$ (the longest relaxation time in our simulations), and calculating the wide-orbit planet occurrence rate of young versus old stars. If massive planets in wide orbits are equally common around young and old stars, gravitational instability is almost certainly their formation mechanism. Any age trend would indicate that planets on wide orbits are transient relics of scattering from the inner disk. In conducting such an experiment, care must be taken to control for the fact that planets cool and become less detectable over time.

Further evidence for dual modes of planet formation, with core accretion operating near the star and gravitational instability dominant at large distance, would come from examining the occurrence ratio of long-period to short-period gas giants as a function of stellar mass. We predict that this ratio should be highest for M dwarfs, for which core accretion is inefficient, short-period planets are rare (Johnson et al. 2007; Cumming et al. 2008), and disk fragmentation appears to produce objects of planetary mass (Clarke 2009). Note, however, that preferred fragment mass in extended disks is still a subject of considerable debate (e.g., Boley et al. 2009; Kratter et al. 2009). Core accretion on its own would produce a planet population where the occurrence ratio of long-period to short-period gas giants has the opposite trend, increasing with stellar mass.

As further evidence for gravitational instability, we note that HR 8799 is a $\lambda$ Bootis star, a metal-poor Pop I star that probably accreted a thin layer of low-metallicity gas after formation (Marois et al. 2008). Even if the protoplanetary disk surrounding HR 8799 was not originally massive, passing through a nearby cloud could have triggered Bondi-Hoyle accretion onto the disk and destabilized it (Throop \& Bally 2008).

One further discriminant of planets formed by disk instability and core accretion/scattering is near-infrared color. Fortney et al. (2008) find that model planets with the $\sim 5 \times$ solar metallicity of Jupiter and Saturn-generally interpreted as a signature of core accretion-are redder by $1.5 \mathrm{mag}$ in $H-K$ than solar-metallicity models. Note, however, the simulations by Helled \& Schubert (2008) showing that planets formed by disk instability may later be enriched by planetesimal capture.

Finally, direct detection of planets is still a young enough field that finding as many planets as possible, without regard to the properties of their host stars, is a worthwhile goal. Gravitational instability naturally produces planets on wide orbits (20-150 AU, Mayer et al. 2004; Boley 2009; Clarke 2009; Rafikov 2009; Stamatellos \& Whitworth 2009), but the core accretion zone of proto-A stars extends to $35 \mathrm{AU}$ and can be directly imaged for nearby targets. Our work confirms the finding of Kennedy \& Kenyon (2008) that A stars have wider planet formation zones than lower-mass stars. Lovis \& Mayor (2007) also found the highest average planet mass around A stars versus $G$ and $M$ stars, which aids planet detectability. Furthermore, A star disks experience the highest infall rates during formation and are better candidates than lower-mass $G$ or M stars for triggered gravitational instability (Kratter et al. 2009). We do not believe it is a coincidence that HR 8799 and Fomalhaut are both A stars. Where the goal is simply to image 
planets, A stars make the best targets. Note that here we are distinguishing between the bulk planet detection likelihoodwhich so far is highest for A stars in both radial velocity and direct imaging surveys-and the ratio of long-period to shortperiod planet occurrence.

Funding for S.D.R.'s work was provided by NASA through the Spitzer Space Telescope Fellowship Program. E.B.F. and D.V. received support from the National Science Foundation under the NSF grant listed below and the University of Florida under the auspices of U.F.'s High-Performance Computing Center. This project was the outgrowth of discussions at the 2009 Florida Astrophysics Winter Workshop which was supported by the University of Florida and the NSF grant listed below. The authors acknowledge valuable discussion among the workshop participants, particularly Ruth Murray-Clay, Kaitlin Kratter, Althea Moorhead, and Andrew Youdin. The authors also thank Aaron Boley, John Johnson, Christian Marois, Greg Laughlin, and Peter Bodenheimer for input on this work. The referee, Richard Durisen, provided particularly valuable insight into the current state of the gravitational instability subfield. This material is based upon work supported by the National Science Foundation under grant No. 0707203.

\section{REFERENCES}

Adams, F. C., Ruden, S. P., \& Shu, F. H. 1989, ApJ, 347, 959

Andrews, S. M., \& Williams, J. P. 2007, ApJ, 671, 1800

Barnes, R., Quinn, T. R., Lissauer, J. J., \& Richardson, D. C. 2009, Icarus, 203, 626

Boley, A. C. 2009, ApJ, 695, 53

Boley, A. C., \& Durisen, R. H. 2008, ApJ, 685, 1193

Boley, A. C., Durisen, R. H., Nordlund, Å., \& Lord, J. 2007, ApJ, 665, 1254

Boley, A. C., Hayfield, T., Mayer, L., \& Durisen, R. H. 2009, Icarus, submitted (arXiv:0909.4543)

Boss, A. P. 2000, ApJ, 536, L101

Boss, A. P. 2005, ApJ, 629, 535

Boss, A. P. 2006, ApJ, 637, L137

Boss, A. P. 2007, ApJ, 661, L73

Butler, R. P., et al. 2006, ApJ, 646, 505

Cai, K., Durisen, R. H., Boley, A. C., Pickett, M. K., \& Mejía, A. C. 2008, ApJ, 673,1138

Cai, K., Durisen, R. H., Michael, S., Boley, A. C., Mejía, A. C., Pickett, M. K., \& D'Alessio, P. 2006, ApJ, 636, L149

Cai, K., Pickett, M. K., Durisen, R. H., \& Milne, A. M. 2009, ApJ, submitted (arXiv:0907.4213)

Cha, S.-H., \& Whitworth, A. P. 2003, MNRAS, 340, 91

Chambers, J. E. 1999, MNRAS, 304, 793

Chatterjee, S., Ford, E. B., Matsumura, S., \& Rasio, F. A. 2008, ApJ, 686, 580

Chen, C. H., Sheehan, P., Watson, D. M., Manoj Puravankara, P., \& Najita, J. R. 2009, ApJ, 701, 1367

Chiang, E., \& Goldreich, P. 1997, ApJ, 490, 368

Chiang, E. I., Joung, M. J., Creech-Eakman, M. J., Qi, C., Kessler, J. E., Blake, G. A., \& van Dishoeck, E. F. 2001, ApJ, 547, 1077

Clarke, C. J. 2009, MNRAS, 396, 1066

Clark, P. C., \& Bonnell, I. A. 2005, MNRAS, 361, 2

Cumming, A., Butler, R. P., Marcy, G. W., Vogt, S. S., Wright, J. T., \& Fischer, D. A. 2008, PASP, 120, 531

Currie, T., Lada, C. J., Plavchan, P., Robitaille, T. P., Irwin, J., \& Kenyon, S. J. 2009, ApJ, 698, 1

D’Antona, F., \& Mazzitelli, I. 1994, ApJS, 90, 467

Dodson-Robinson, S. E., \& Bodenheimer, P. 2009, ApJ, 695, L159

Dodson-Robinson, S. E., Bodenheimer, P., Laughlin, G., Willacy, K., Turner, N. J., \& Beichman, C. A. 2008, ApJ, 688, L99

Dodson-Robinson, S. E., Willacy, K., Bodenheimer, P., Turner, N. J., \& Beichman, C. A. 2009, Icarus, 200, 672

Durisen, R. H., Boss, A. P., Mayer, L., Nelson, A. F., Quinn, T., \& Rice, W. K. M. 2007, in Protostars and Planets V, ed. B. Reipurth, D. Jewitt, \& K. Keil (Tucson, AZ: Univ. Arizona Press), 607

Fabrycky, D. C., \& Murray-Clay, R. A. 2008, ApJ, submitted (arXiv:0812.0011)
Fischer, D. A., \& Valenti, J. 2005, ApJ, 622, 1102

Ford, E. B., \& Rasio, F. A. 2008, ApJ, 686, 621

Forgan, D., Rice, K., Stamatellos, D., \& Whitworth, A. 2009, MNRAS, 394, 882

Fortney, J. J., Marley, M. S., Saumon, D., \& Lodders, K. 2008, ApJ, 683, 1104 Gomes, R. S., Morbidelli, A., \& Levison, H. F. 2004, Icarus, 170, 492

Gonzalez, G. 1998, A\&A, 334, 221

Goździewski, K., \& Migaszewski, C. 2009, MNRAS, 397, L16

Greenzweig, Y., \& Lissauer, J. J. 1992, Icarus, 100, 440

Hahn, J. M., \& Malhotra, R. 1999, AJ, 117, 304

Helled, R., \& Schubert, G. 2008, Icarus, 198, 156

Ida, S., \& Lin, D. N. C. 2004, ApJ, 604, 388

Johnson, J. A., Butler, R. P., Marcy, G. W., Fischer, D. A., Vogt, S. S., Wright, J. T., \& Peek, K. M. G. 2007, ApJ, 670, 833

Kalas, P., Graham, J. R., \& Clampin, M. 2005, Nature, 435, 1067

Kalas, P., et al. 2008, Science, 322, 1345

Kary, D. M., \& Lissauer, J. J. 1994, in Numerical Simulations in Astrophysics, ed. J. Franco et al. (Cambridge: Cambridge Univ. Press)

Kennedy, G. M., \& Kenyon, S. J. 2008, ApJ, 673, 502

Kenyon, S. J., \& Bromley, B. C. 2008, ApJS, 179, 451

Kratter, K. M., Matzner, C. D., \& Krumholz, M. R. 2008, ApJ, 681, 375

Kratter, K. M., Matzner, C. D., Krumholz, M. R., \& Klein, R. I. 2009, arXiv:0907.3476

Kratter, K. M., Murray-Clay, R. A., \& Youdin, A. N. 2009, arXiv:0909.2644

Lafreniére, D., Marois, C., Doyon, R., \& Barman, T. 2009, ApJ, 694, 148

Laughlin, G., Bodenheimer, P., \& Adams, F. C. 2004, ApJ, 612, L73

Laughlin, G., \& Korchagin, V. 1996, ApJ, 460, 855

Laughlin, G., Korchagin, V., \& Adams, F. C. 1998, ApJ, 504, 945

Laughlin, G., \& Rozyczka, M. 1996, ApJ, 456, 279

Le Bouquin, J.-B., Absil, O., Benisty, M., Massi, F., Mérand, A., \& Stefl, S. 2009, A\&A, 498, L41

Levison, H. F., \& Stewart, G. R. 2001, Icarus, 153, 224

Lissauer, J. J. 1993, ARA\&A, 31, 129

Lodato, G., \& Rice, W. K. M. 2005, MNRAS, 358, 1489

Lovis, C., \& Mayor, M. 2007, A\&A, 472, 657

Lynden-Bell, D., \& Pringle, J. E. 1974, MNRAS, 168, 603

Lyra, W., Johansen, A., Klahr, H., \& Piskunov, N. 2008, A\&A, 479, 883

Marois, C., Macintosh, B., Barman, T., Zuckerman, B., Song, I., Patience, J. Lafrenière, D., \& Doyon, R. 2008, Science, 322, 1348

Mayer, L., Quinn, T., Wadsley, J., \& Stadel, J. 2004, ApJ, 609, 1045

Mejía, A. C., Durisen, R. H., Pickett, M. K., \& Cai, K. 2005, ApJ, 619, 1098

Mizuno, H. 1980, Prog. Theor. Phys., 64, 544

Morbidelli, A., \& Crida, A. 2007, Icarus, 191, 158

Pepliński, A., Artymowicz, P., \& Mellema, G. 2008, MNRAS, 387, 1063

Pickett, M. K., \& Durisen, R. H. 2007, ApJ, 654, L155

Pickett, B. K., Mejía, A. C., Durisen, R. H., Cassen, P. M., Berry, D. K., \& Link, R. P. 2003, ApJ, 590, 1060

Press, W. H., Teukolsky, S. A., Vetterling, W. T., \& Flannery, B. P. 1992 Numerical Recipes in Fortran 77 (2nd ed.; Cambridge: Cambridge Univ. Press)

Rafikov, R. R. 2005, ApJ, 621, L69

Rafikov, R. R. 2009, ApJ, 704, 281

Raymond, S. N., Armitage, P. J., \& Gorelick, N. 2009, ApJ, 699, 88

Raymond, S. N., Barnes, R., Armitage, P. J., \& Gorelick, N. 2008, ApJ, 687, L107

Reidemeister, M., Krivov, A. V., Schmidt, T. O. B., Fiedler, S., Müller, S., Löhne, T., \& Neuhäuser, R. 2009, A\&A, 503, 247

Rice, W. K. M., Lodato, G., Pringle, J. E., Armitage, P. J., \& Bonnell, I. A. 2004, MNRAS, 355, 543

Robinson, S. E., Laughlin, G., Bodenheimer, P., \& Fischer, D. 2006, ApJ, 643 484

Safronov, V. S. 1969, Evolution of the Protoplanetary Cloud and Formation of the Earth and Planets (in Russian; English translation 1972 NASA-TTF-667 (Moscow: Nauka))

Scharf, C., \& Menou, K. 2009, ApJ, 693, 113L

Shakura, N. I., \& Syunyaev, R. A. 1973, A\&A, 24, 337

Stamatellos, D., \& Whitworth, A. P. 2008, A\&A, 480, 879

Stamatellos, D., \& Whitworth, A. P. 2009, MNRAS, 392, 413

Su, K. Y. L., et al. 2009, ApJ, 705, 314

Throop, H. B., \& Bally, J. 2008, AJ, 135, 2380

Tsiganis, K., Gomes, R., Morbidelli, A., \& Levison, H. F. 2005, Nature, 435, 459

Veras, D., Crepp, J. R., \& Ford, E. B. 2009, ApJ, 696, 1600

Vorobyov, E. I., \& Basu, S. 2006, ApJ, 650, 956

Whitworth, A. P., \& Stamatellos, D. 2006, A\&A, 458, 817

Zuckerman, B., \& Song, I. 2009, A\&A, 493, 1149 